\title{
Longitudinal and vertical focusing with a field gradient spiral inflector
}

\author{
A. H. Barnard๑, ${ }^{*}$ J. I. Broodryk, J. L. Conradie, J. G. de Villiers®, J. P. Mira®, \\ F. Nemulodi $\odot$, and R. Thomae \\ iThemba Laboratory for Accelerator Based Sciences, Faure Road, Cape Town 7600, South Africa
}

(Received 12 October 2020; accepted 19 January 2021; published 5 February 2021)

\begin{abstract}
Traditional spiral inflectors of the Belmont-Pabot type are commonly used for axial injection of external ion beams into cyclotrons. These inflectors are designed to control the trajectory of the central path, and do not actively focus the beam in the vertical and longitudinal directions. This can introduce effects such as a large vertical divergence and a debunching longitudinal spread, making it difficult to match the injection line emittance to the cyclotron acceptance. In an attempt to overcome this, some recent inflectors have started incorporating electrodes specially shaped to produce field gradients along the central path, thereby influencing the inflector optics. This method has shown some success, and at iThemba LABS an inflector was built exhibiting good vertical focusing. However, it performed poorly longitudinally, worse than traditional spiral inflectors. In this article a generalized field gradient spiral inflector design is presented, based on a mathematical description of all possible first-order field gradients along the central path. Such a design is numerically optimized to simultaneously focus longitudinally and vertically. Experimental studies of this design show a $60 \%$ improvement in overall current extracted from the cyclotron.
\end{abstract}

DOI: 10.1103/PhysRevAccelBeams.24.023501

\section{INTRODUCTION}

Introduced by Belmont and Pabot in 1966, traditional spiral inflectors are used to inject external axial beams into the midplane of a cyclotron. This is done by applying an electric field perpendicular to the central beam path in the presence of the main magnetic field, in such a way that the beam travels in a spiraling motion and is eventually bent from its axial direction to the central acceleration plane [1].

Unfortunately, spiral inflectors can defocus and spread out the beam, so that it is poorly matched to the cyclotron acceptance [2]. The transverse and longitudinal 2D emittances are also not necessarily conserved anymore, due to the complex coupling of motion in all directions. The full $6 \mathrm{D}$ emittance is conserved, but this does not in general limit the size of the projected 2D emittances [3].

An example of this beam degradation is the large vertical divergence typically found at the inflector exit, as shown in Fig. 1, which has been observed to lead to beam loss in the inner part of the cyclotron. In the Solid Pole Cyclotron 2 (SPC2) at iThemba LABS, numerical modeling of the vertical motion produced by the Belmont-Pabot inflector

\footnotetext{
*hbarnard@tlabs.ac.za

Published by the American Physical Society under the terms of the Creative Commons Attribution 4.0 International license. Further distribution of this work must maintain attribution to the author(s) and the published article's title, journal citation, and DOI.
}

shows a beam loss of $30 \%-40 \%$ even before reaching the first acceleration gap, due to vertical losses on a collimator.

To address this problem of vertical defocusing, one possible solution is to add a focusing element such as an electric or magnetic quadrupole immediately downstream of the inflector [4]. But this is often difficult to do, since there is very limited space available in the central region and it also does not address the emittance blowup due to the coupled motion inside the inflector. Another method is to incorporate the focusing into the inflector itself, by introducing electric field gradients along the central trajectory. One such design, developed at Dubna [2], gives the electrodes a V shape in the transverse plane, as shown in Fig. 2, and is similar in nature to the vertical focusing produced in a spherical electrostatic bend. Another design, from Sumitomo Heavy Industries [5], uses angled electrodes in the transverse plane, as shown in Fig. 2, and also modifies the entrance and exit edge angles to generate quadrupoles in the fringe field region.

Recently at iThemba LABS a new inflector was developed that also utilized angled electrodes and edge effects to obtain very good vertical focusing, resulting in an experimentally measured $100 \%$ improvement in the extracted current from the cyclotron, whenever the buncher was not used [6]. However, this inflector also introduced an unwanted longitudinal spread, which greatly decreased its time-dependent performance, so that the improvement in transmission dropped to around $20 \%$ when the buncher was activated. 


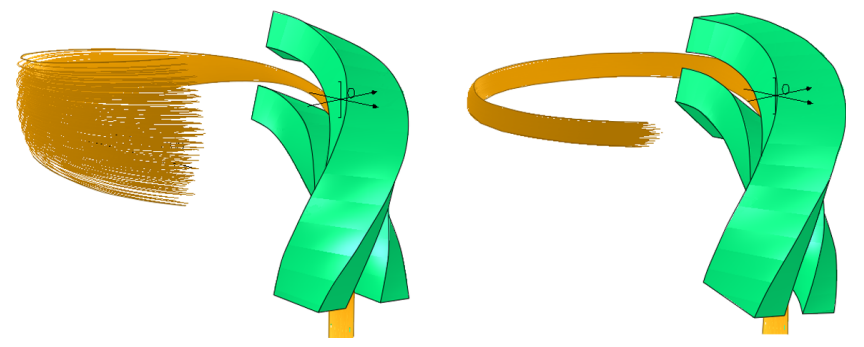

FIG. 1. A traditional spiral inflector (left) showing large vertical divergence, and a field gradient spiral inflector (right) with much improved vertical behavior.
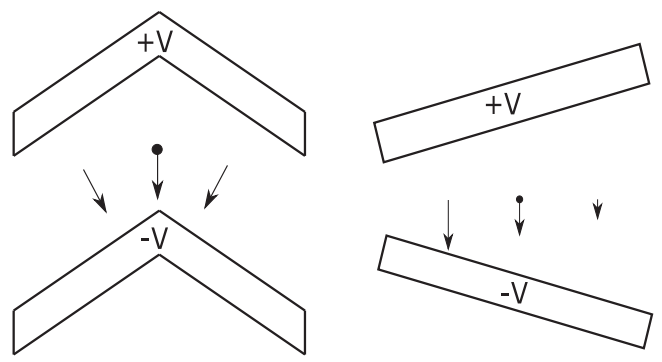

FIG. 2. Electrode cross sections in the transverse plane: The Dubna V shape (left) and the Sumitomo angled electrodes (right).

This focused our attention on the longitudinal influence of inflectors, a topic that has received less attention in the past. In spiral inflectors initial transverse displacements at the inflector entrance can result in final longitudinal displacements: $(\ell \mid x),\left(\ell \mid x^{\prime}\right),(\ell \mid y),\left(\ell \mid y^{\prime}\right) \neq 0$. This has the effect of longitudinally elongating a bunch of particles that all arrive at the inflector at the same time, thereby producing a debunching effect. This effect is very significant and TOSCA [7] models of the traditional spiral inflector used at iThemba LABS show a $\pm 39^{\circ}$ rf phase spread when injected with a typical beam of $90 \pi \mathrm{mm} \mathrm{mrad}$ transverse emittance and no momentum spread. This shows that an inflector should ideally focus both longitudinally and vertically at the same time.

\section{THE SPC2 CYCLOTRON AND C-INFLECTOR}

The work described in this article was performed on the Solid Pole Cyclotron 2 (SPC2), a $K=11$ injector cyclotron for the main $K=200$ Separated Sector Cyclotron at iThemba LABS. SPC2 is capable of accelerating particles along three different orbit trajectories, and heavy ions are injected into an eight turn orbit geometry using the spiral inflector called inflector-C [8]. For this orbit geometry an rf harmonic number $h=6$ is used. In order to distinguish between the different inflectors described here, the chronological names $\mathrm{C} 1, \mathrm{C} 2$ and $\mathrm{C} 3$ are used. Inflector-C1 is the original Belmont-Pabot design, inflector-C2 is a field gradient design producing very strong vertical focusing,
TABLE I. Belmont-Pabot parameters used by the C-inflectors.

\begin{tabular}{lcl}
\hline \hline Magnetic bending radius & $R_{m}$ & $4.9 \mathrm{~cm}$ \\
Electric bending radius & $A$ & $6.0 \mathrm{~cm}$ \\
Tilt parameter & $k^{\prime}$ & 0.38 \\
\hline \hline
\end{tabular}

and inflector-C3 is a more general field gradient design capable of simultaneous vertical and longitudinal focusing. The Belmont-Pabot design parameters for the central trajectory in these inflectors are shown in Table I.

\section{COORDINATE SYSTEM}

To aid in the description of the inflectors, an overview of the optical $\left(u_{r}, h_{r}, s\right)$ spiral inflector coordinate system is given here, as shown in Fig. 3 [9]. The location of the central path is indicated by $\mathbf{x}_{0}(s)$ where $s$ is the path length, and the direction of motion is given by the unit vector $\hat{s}=\mathbf{x}_{0}^{\prime}$ where the dash indicates differentiation with respect to the path length. At every point along the path a transverse $\left(u_{r}, h_{r}\right)$ plane is located perpendicular to $\hat{s}$, so that $\hat{u}_{r}$ points along the direction of the electric field, and $\hat{h}_{r}$ is perpendicular to the field, so that $\left(\hat{u}_{r}, \hat{h}_{r}, \hat{s}\right)$ forms a right-handed system. The location of a point in space is given by

$$
\mathbf{x}\left(u_{r}, h_{r}, s\right)=\mathbf{x}_{0}(s)+u_{r} \hat{u}_{r}(s)+h_{r} \hat{h}_{r}(s) .
$$

The use of the " $r$ " subscript is for historical reasons, to distinguish this rotated frame aligned with the electric field from an unrotated $(\hat{u}, \hat{h}, \hat{s})$ system where $\hat{h}$ is always horizontal. During the design of the electrodes the rotated reference frame is used since it provides an easier way of describing the electric field and its gradients. On the other hand, when calculating the transfer matrices the unrotated frame is used, since it aligns with the horizontal and vertical directions. In order to work properly with the coordinate system, it is useful to describe the motion of the orthonormal $\left(\hat{u}_{r}, \hat{h}_{r}, \hat{s}\right)$ frame by a rotation around a curvature vector $\boldsymbol{\kappa}(s)$ :
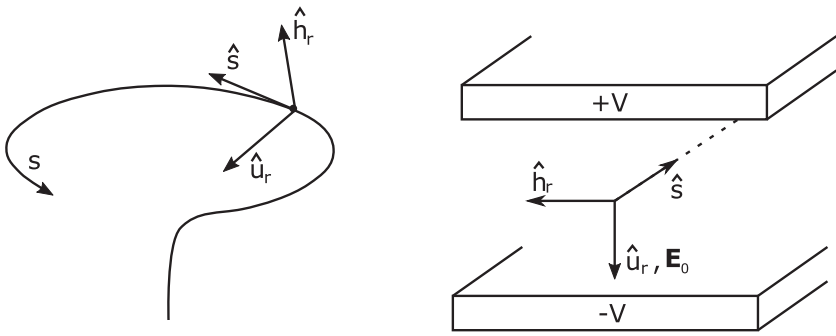

FIG. 3. Optical coordinate system around the central path (left). Electrode cross section of a Belmont-Pabot inflector in the transverse $\left(u_{r}, h_{r}\right)$ plane (right). 


$$
\begin{gathered}
\hat{u}_{r}^{\prime}=\kappa_{s} \hat{h}_{r}-\kappa_{h} \hat{s} \\
\hat{h}_{r}^{\prime}=\kappa_{u} \hat{s}-\kappa_{s} \hat{u}_{r} \\
\hat{s}^{\prime}=\kappa_{h} \hat{u}_{r}-\kappa_{u} \hat{h}_{r} .
\end{gathered}
$$

The basis vectors of the $\left(u_{r}, h_{r}, s\right)$ coordinate system are indicated by

$$
\begin{gathered}
\mathbf{b}_{1}=\frac{\partial \mathbf{x}}{\partial u_{r}}=\hat{u}_{r} \\
\mathbf{b}_{2}=\frac{\partial \mathbf{x}}{\partial h_{r}}=\hat{h}_{r} \\
\mathbf{b}_{3}=\frac{\partial \mathbf{x}}{\partial s}=\hat{s}\left(1-u_{r} \kappa_{h}+h_{r} \kappa_{u}\right)+\kappa_{s}\left(u_{r} \hat{h}_{r}-h_{r} \hat{u}_{r}\right) .
\end{gathered}
$$

This shows that the basis vectors are not always orthogonal, except on the central trajectory itself. The dual basis vectors, indicated by $\mathbf{b}^{i}$, are defined according to

$$
\mathbf{b}^{i} \cdot \mathbf{b}_{j}=\delta_{j}^{i}
$$

which means

$$
\begin{aligned}
\mathbf{b}^{1} & =\hat{u}_{r}+\frac{h_{r} \kappa_{s}}{1-u_{r} \kappa_{h}+h_{r} \kappa_{u}} \hat{s} \\
\mathbf{b}^{2} & =\hat{h}_{r}-\frac{u_{r} \kappa_{s}}{1-u_{r} \kappa_{h}+h_{r} \kappa_{u}} \hat{s} \\
\mathbf{b}^{3} & =\frac{1}{1-u_{r} \kappa_{h}+h_{r} \kappa_{u}} \hat{s} .
\end{aligned}
$$

All of these terms are known analytically for a traditional Belmont-Pabot inflector.

\section{INFLECTOR WITH VERTICAL FOCUSING}

The C2-inflector was developed at iThemba LABS for the express purpose of vertical focusing [6]. It used electrodes angled towards each other, similar to the Sumitomo design in Fig. 2, as well as angled entrance and exit electrode edges, to produce quadrupole fields of the type $\partial E_{u_{r}} / \partial h_{r}, \partial E_{h_{r}} / \partial u_{r}$.

The results of experimental testing of the $\mathrm{C} 2$-inflector are shown in Fig. 4. With the buncher turned off, the inflector performed very well, roughly doubling the extracted current. With the buncher turned on, the beam is still injected very well, but the extraction sees a large drop in performance.

This decrease in performance is thought to be the result of a large longitudinal spread introduced by $\mathrm{C} 2$, which translates into a large energy spread during the acceleration process, resulting in a broad beam with poor extraction

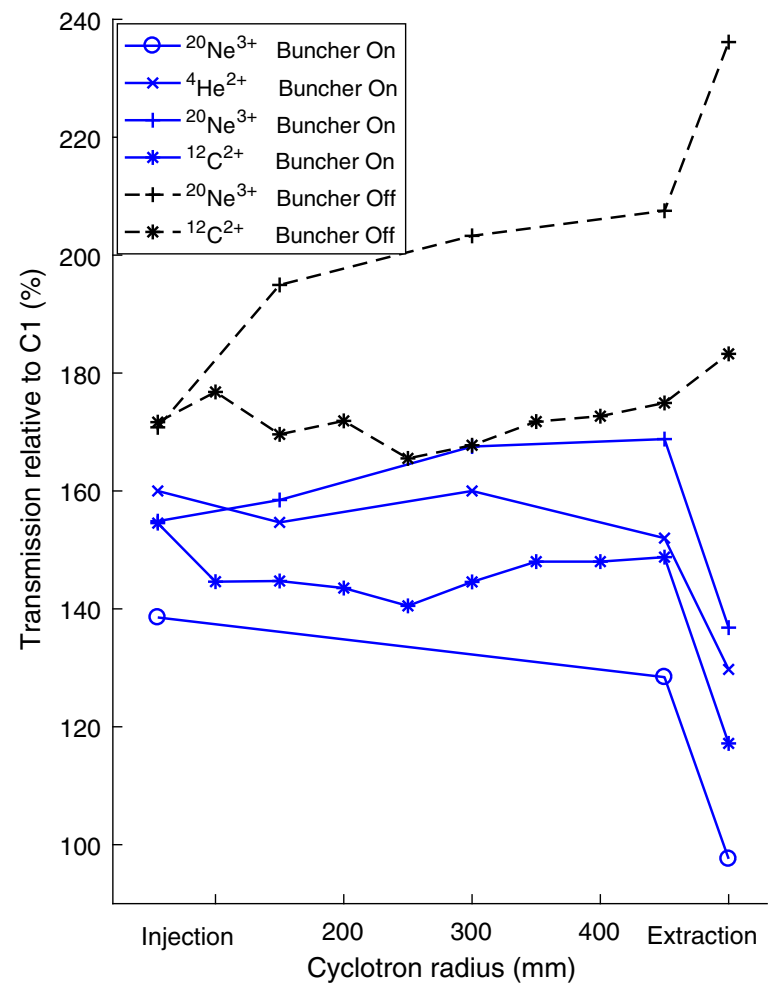

FIG. 4. Experimental transmission through SPC2 when using the vertically focusing $\mathrm{C} 2$-inflector, as a percentage of the standard C1-inflector transmission. Solid lines are with the buncher and dotted lines are without the buncher.

efficiency. When $\mathrm{C} 2$ is injected with a very short bunch, of negligible longitudinal and momentum spread, coming from a typical beam of $90 \pi \mathrm{mm}$ mrad transverse emittance, TOSCA models show a severe $\pm 13 \mathrm{~mm}$ longitudinal spread $\left( \pm 90^{\circ}\right.$ rf phase $)$ at the first acceleration gap. This debunching effect is made worse by the high harmonic number $(h=6)$ rf frequency used by SPC2, which reduces the rf phase acceptance.

Attempts at improving the longitudinal spread of a C2type design have proven unsuccessful. Extra degrees of design freedom were required, and so it was necessary to consider all possible field gradients in more detail.

\section{FIRST ORDER OPTICS: ELECTRIC FIELD GRADIENTS}

The first order optics of a system can be determined by knowing the electric and magnetic fields on the central path, as well as their first order gradients. In a traditional Belmont-Pabot inflector the magnetic gradient is assumed to be zero, and the electrodes are placed parallel to each other, in order to keep the electric field as uniform as possible. There are however several ways of intentionally creating electric gradients by shaping the electrodes. For illustrative purposes the 3D curvature of the central trajectory can be neglected, and the electric field 
approximated as purely $2 \mathrm{D}$ in the transverse plane. A normal quadrupole field can then be created by using hyperbola-shaped electrodes, as shown on the left in Fig. 5:

$$
\frac{\partial E_{u_{r}}}{\partial h_{r}}-\frac{\partial E_{h_{r}}}{\partial u_{r}}=0 .
$$

Alternatively a skew quadrupole can be created by using concentric electrodes, as shown on the right in Fig. 5:

$$
\frac{\partial E_{u_{r}}}{\partial u_{r}}+\frac{\partial E_{h_{r}}}{\partial h_{r}}=0 .
$$

Fringe fields at the electrode edges affect the inflector in important ways. At the entrance and exit the fringe field has large gradients, which can be harnessed to produce quadrupoles along the beam path by reorienting the electrode face. When the edge is cut at an angle to the beam path in the $\left(h_{r}, s\right)$ plane, as shown on the left in Fig. 6, a normal quadrupole is produced in the fringe field region. The edge can also be cut at an angle in the $\left(u_{r}, s\right)$ plane, shown on the right in Fig. 6. This produces field gradients, but it also violates the design condition of $\phi=0$ on the central path. This is however a situation that occurs frequently in actual inflectors, where the electrodes are
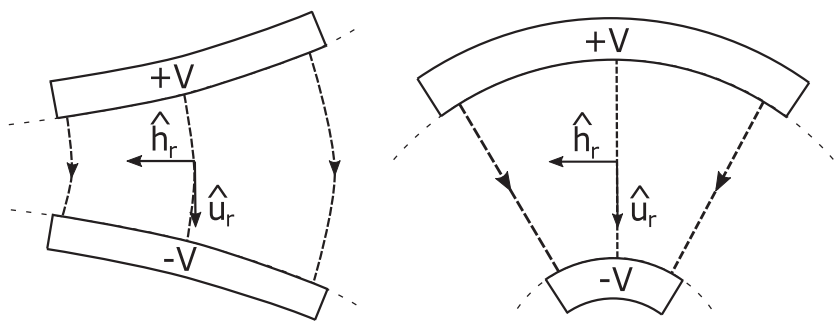

FIG. 5. Creating normal quadrupole field gradients by using hyperbolic electrodes (left), and creating skew quadrupole field gradients by using cylindrical electrodes (right). The left-hand method is similar to the angled electrodes used by Sumitomo and by $\mathrm{C}$, while the right-hand method is similar to the V-shaped Dubna design shown in Fig. 2.
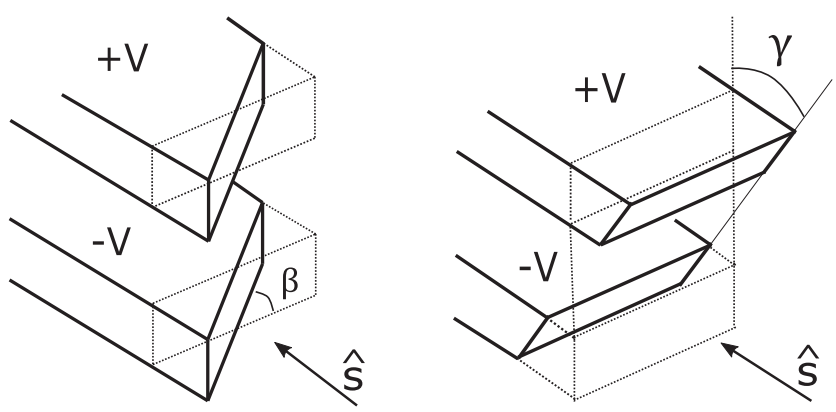

FIG. 6. Creating transverse field gradients in the fringe field region by cutting both electrodes in the $\left(h_{r}, s\right)$ plane (left) and in the $\left(u_{r}, s\right)$ plane (right). sometimes shortened to compensate for the fringe field's bending of the central path.

Fringe fields are also present along the sides of the inflector, at the $\pm h_{r}$ edges, and these fields may produce quadrupoles on the central path. This effect is exploited by work at Dubna [10], where the positive and negative electrodes are kept parallel, but given different widths, producing skew quadrupoles on the central path. In the design of the $\mathrm{C} 2$ and $\mathrm{C} 3$ inflectors presented here, the electrodes lie on equipotential surfaces, and the fringe fields are regarded as unwanted side effects introduced by the finite electrode width. These fringe quadrupoles can be reduced by using electrodes with a sufficiently large widthto-gap ratio.

\section{A MATHEMATICAL DESCRIPTION}

In order to combine these methods of gradient creation, it is useful to try to find the most general format of the electrical potential in the region close to the central path, and to calculate the electric fields and field gradients from this potential. Obtaining an analytic expression for the electric fields in this way has been suggested in [11], but the approach here is more general, in that the full tensor expression for the Laplacian is used. This is advisable for higher order solutions, since the curvilinear $\left(u_{r}, h_{r}, s\right)$ coordinate system is not locally orthogonal, except on the central path. In such a general case the Laplacian becomes [12]:

$$
\nabla^{2} \phi=\frac{1}{\sqrt{g}} \sum_{i} \frac{\partial}{\partial u^{i}}\left(\sqrt{g} \sum_{j} \frac{\partial \phi}{\partial u^{j}} g^{i j}\right)=0,
$$

where $u^{1}, u^{2}, u^{3}$ refer to the coordinate values $u_{r}, h_{r}, s$ respectively. The matrix $g^{i j}$ is the inverse of the metric matrix $g_{i j}$ and $g$ refers to the determinant of the metric matrix:

$$
g_{i j}=\mathbf{b}_{i} \cdot \mathbf{b}_{j}=\left[\begin{array}{ccc}
1 & 0 & -h_{r} \kappa_{s} \\
0 & 1 & u_{r} \kappa_{s} \\
-h_{r} \kappa_{s} & u_{r} \kappa_{s} & K
\end{array}\right],
$$

where

$$
K=\kappa_{s}^{2}\left(u_{r}^{2}+h_{r}^{2}\right)+\left(1-u_{r} \kappa_{h}+h_{r} \kappa_{u}\right)^{2} .
$$

The complicated expression for the Laplacian reduces to a simpler form in the region close to the central path where $u_{r}, h_{r} \rightarrow 0$. Representing all the first and higher order terms of $u_{r}$ and $h_{r}$ by $\mathcal{O}\left(u_{r}, h_{r}\right)$, we see

$\frac{\partial^{2} \phi}{\partial u_{r}^{2}}+\frac{\partial^{2} \phi}{\partial h_{r}^{2}}+\frac{\partial^{2} \phi}{\partial s^{2}}+\kappa_{u} \frac{\partial \phi}{\partial h_{r}}-\kappa_{h} \frac{\partial \phi}{\partial u_{r}}=\mathcal{O}\left(u_{r}, h_{r}\right)$.

Note that by design $\phi=0$ on the central path, so $\phi^{\prime \prime}$ is first or higher order in $u_{r}, h_{r}$ and can also be grouped inside $\mathcal{O}\left(u_{r}, h_{r}\right)$. Since we are only interested in the first order 
optics, which is determined by the first order electric fields, a second order expression for $\phi$ can be substituted into this equation. Keeping only the lowest order terms in $u_{r}, h_{r}$ we then obtain

$$
\frac{\partial^{2} \phi}{\partial u_{r}^{2}}+\frac{\partial^{2} \phi}{\partial h_{r}^{2}}+\hat{s}^{\prime} \cdot \mathbf{E}_{0}=0
$$

At a given $s$, this becomes the 2D Poisson equation in the $\left(u_{r}, h_{r}\right)$ plane, with a constant forcing term. The potential and the electric field on the central path are known, giving the following format as the most general expression for the potential:

$\phi=-u_{r} E_{0}-Q_{1} E_{0} \frac{u_{r}^{2}-h_{r}^{2}}{2}-Q_{2} E_{0} u_{r} h_{r}-\frac{u_{r}^{2}}{2} \hat{s}^{\prime} \cdot \mathbf{E}_{0}$,

where $E_{0}$ and $\hat{s}^{\prime} \cdot \mathbf{E}_{0}$ are known functions of the central path, so that the quadrupole parameters $Q_{1}(s)$ and $Q_{2}(s)$ are the only free terms, and can be selected by the inflector designer. The $Q_{1}$ term corresponds to the spherical electrodes in Fig. 5, and the $Q_{2}$ terms correspond to the angled electrodes. These definitions of $Q_{1}$ and $Q_{2}$ have been selected so that a traditional Belmont-Pabot inflector with its parallel electrodes should have $Q_{1}=Q_{2}=0$. Note that in our previous publication [6] on the $\mathrm{C} 2$-inflector a single quadrupole parameter $Q(s)$ was used to describe the angled electrodes, which corresponds to $Q=-Q_{2}$ in this work.

A desired potential can then be physically obtained by constructing the inflector electrodes so they lie on equipotential surfaces. Since Eq. (13) only holds at small values of $u_{r}, h_{r}$ it is not applicable to the electrode surfaces at the entrance and exit, and we limit its use to the interior of the inflector. Care should also be taken that $Q_{1}(s)$ and $Q_{2}(s)$ vary smoothly, since their derivatives contribute to the $\mathcal{O}\left(u_{r}, h_{r}\right)$ terms, and thereby reduce the $\left(u_{r}, h_{r}\right)$ region within which $\mathcal{O}\left(u_{r}, h_{r}\right)$ may be neglected.

The electric field is obtained from the gradient of the potential:

$$
\mathbf{E}=-\nabla \phi=-\sum_{i} \frac{\partial \phi}{\partial u^{i}} \mathbf{b}^{i}
$$

When writing the electric field in component form,

$$
\mathbf{E}=E_{u_{r}} \hat{u}+E_{h_{r}} \hat{h}+E_{s} \hat{s},
$$

it is found that the components are given, to first order in $u_{r}$, $h_{r}$, by

$$
\begin{gathered}
E_{u_{r}}=E_{0}+u_{r}\left(Q_{1} E_{0}+\hat{s}^{\prime} \cdot \mathbf{E}_{0}\right)+h_{r} Q_{2} E_{0} \\
E_{h_{r}}=u_{r} Q_{2} E_{0}-h_{r} Q_{1} E_{0} \\
E_{s}=u_{r} E_{0}^{\prime}+h_{r} \kappa_{s} E_{0} .
\end{gathered}
$$

\section{NUMERICAL VERIFICATION}

The expression for the electric field derived above, and the method of using the equipotential surfaces as electrodes, can be checked by numerical modeling with TOSCA. As an example given here, the field was computed for an inflector with complicated designer $Q$-values, shown in Fig. 7.
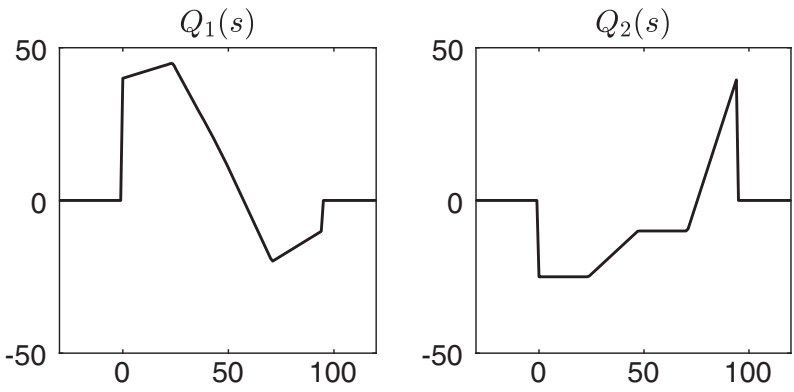

FIG. 7. The designer $Q_{1}$ and $Q_{2}$ (in $\mathrm{m}^{-1}$ ) versus the path length (in $\mathrm{mm}$ ) as used in the verification test. These curves have been chosen arbitrarily and are piecewise smooth inside the inflector.
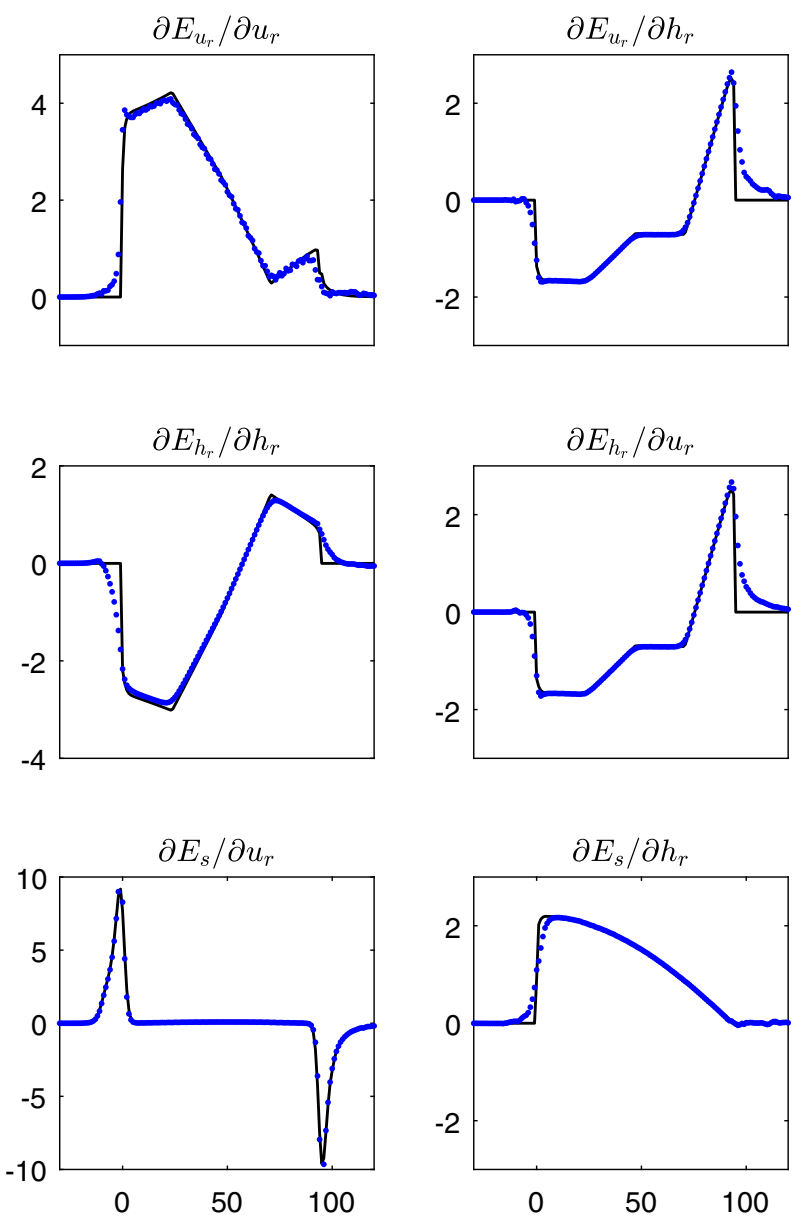

FIG. 8. Electric field gradients (in $10^{7} \mathrm{Vm}^{-1}$ ) along the path length of the inflector (in $\mathrm{mm}$ ), obtained from a 3D TOSCA model. The dotted lines are the numerical field gradients, and the solid lines are the gradients calculated according to Eq. (16). 
The field gradients are shown in Fig. 8, where the numerical values from TOSCA are compared to the values calculated from Eq. (16), using the TOSCA value for $E_{0}(s)$ and the designer $Q$-values. There is good agreement, and the only noticeable discrepancies are at the inflector entrance and exit, where the $Q$-values make discontinuous jumps.

In this test an electrode width of $20 \mathrm{~mm}$ and a nominal electrode gap of $6 \mathrm{~mm}$ were used, which provides a sufficient width-to-gap ratio for ignoring the fringe fields along the $\pm h_{r}$ edges. Furthermore, the design does not contain any entrance and exit edge angles $(\beta=\gamma=0$ in Fig. 6). When edge angles are included, or when the fringe fields play a significant role, the electric field equation is found to remain valid around the zero-potential line, but with modified values of $Q_{1}$ and $Q_{2}$.

\section{OPTIMIZATION PROCEDURE}

The first order inflector optics is controlled by selecting appropriate field gradient parameters: $Q_{1}(s), Q_{2}(s)$ and the entrance and exit face angles $\beta_{1}$ and $\beta_{2}$. The dependence of the inflector's transfer function on the field gradients [write this as $R\left(Q_{1}, Q_{2}, \beta_{1}, \beta_{2}\right)$, where $R$ is the transfer function] is computed numerically. This is performed by a MATLAB program that makes use of a limited number of precalculated 3D electric field solutions obtained with TOSCA, and scaling and extrapolating them linearly to obtain estimates of the electric fields for various inflector geometries at run time, as described in more detail in [6]. Ray tracing is employed, starting at a point well upstream of the inflector and ending at the first acceleration gap. The magnetic field is based on a TOSCA model of the full yoke geometry [13]. The program is able to compute a transfer matrix by means of ray tracing within a few seconds, whereas the full TOSCA-based process would take at least an hour.

To perform the optimization, $Q_{1}(s), Q_{2}(s)$ had to be discretized, and they were represented by piecewise linear functions. As a shorthand notation, we write the end points of these piecewise functions with $Q_{i j}=Q_{i}\left(s_{j}\right)$ where $j=1,2$, 3. A finer discretization can be used in future work, but having three values per function is adequate for producing a rough doublet or a triplet shape. The values of the gradient parameters were then selected by numerically optimizing the transfer matrix,

$$
R\left(Q_{11}, Q_{12}, Q_{13}, Q_{21}, Q_{22}, Q_{23}, \beta_{1}, \beta_{2}\right),
$$

with respect to some scoring function corresponding to the desired focusing qualities. Various different optimization methods were tried, but in the end a randomized sampling to find promising starting points was followed by a steepest descent method to find the local optima. Each electrode surface also had to be evaluated to make sure it had a minimum electrode gap, and did not have a shape that is very difficult to construct.

\section{INFLECTOR WITH LONGITUDINAL AND VERTICAL FOCUSING}

Ideally the inflector should match the injection line emittance to the accelerator acceptance. In the case of the SPC2 however, neither of these properties was known with great certainty. So instead of attempting to match the emittance to the acceptance, it was decided to aim for an inflector design which results in a beam with smaller vertical emittance and a shorter longitudinal spread than the original $\mathrm{C} 1$ inflector. The input beam for this optimization task was assumed to have a $3 \mathrm{~mm} \times 30 \mathrm{mrad}$ elliptical transverse profile and a $0.5 \%$ momentum spread. From the simulations it was found that a design with vertical emittance $20 \%$ less than $\mathrm{C} 1$, and longitudinal spread $45 \%$ less than $\mathrm{C} 1$ was obtainable. If accurate information about the SPC2 acceptance had been available then a better informed design could perhaps have been found, but the aim here was mainly to demonstrate the capability of simultaneous vertical and longitudinal focusing. The simulated 2D phase space profiles of all the inflectors are shown in Fig. 9 and Table II provides a comparison of their performance.

The $Q$-values for the inflectors, as obtained from 3D TOSCA models, are shown in Fig. 10. In accordance with
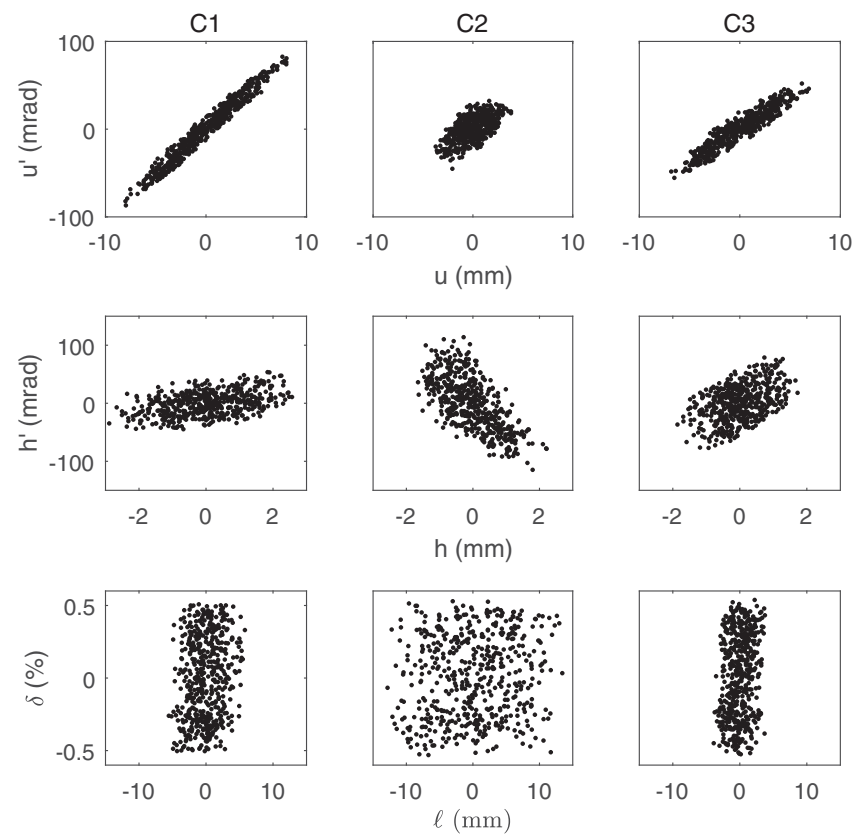

FIG. 9. Phase-space plots of the beam at the first acceleration gap for inflectors C1 (left column), C2 (middle column) and C3 (right column). The top row is vertical $\left(u, u^{\prime}\right)$ space, the middle row is horizontal $\left(h, h^{\prime}\right)$ space and the bottom row is longitudinal $(\ell, \delta)$ space. The $\mathrm{C} 2$-inflector has greatly improved vertical behavior compared to the original $\mathrm{C} 1$ inflector, but a much larger longitudinal spread. The C3 inflector has moderately improved vertical behavior compared to $\mathrm{C} 1$ as well as a smaller longitudinal spread. 
TABLE II. Beam parameters at the first acceleration gap as calculated using TOSCA, based on a $3 \mathrm{~mm} \times 30 \mathrm{mrad}$ beam with $0.5 \%$ momentum spread. The emittances are percentages of the incoming beam.

\begin{tabular}{lccc}
\hline \hline & $\mathrm{C} 1$ & $\mathrm{C} 2$ & $\mathrm{C} 3$ \\
\hline Vertical emittance & 145 & 105 & 120 \\
Vertical half width (mm) & 8.9 & 2.9 & 7.0 \\
Horizontal emittance & 145 & 130 & 120 \\
Horizontal half width (mm) & 3.0 & 1.5 & 2.0 \\
Longitudinal half width (mm) & 5.5 & 12.9 & 3.1 \\
rf phase spread (degrees) & \pm 39 & \pm 90 & \pm 22 \\
\hline \hline
\end{tabular}

expectations both $Q_{1}$ and $Q_{2}$ are very small for the traditional Belmont-Pabot inflector $\mathrm{C} 1$. The $\mathrm{C} 2$-inflector has been designed to have a large value of $Q_{2}$, and this is observed, especially near the entrance and exit, where $\beta$ contributes. The C3-design is the only inflector incorporating both $Q_{1}$ and $Q_{2}$.

During the design process of $\mathrm{C} 3$ it was found that excessive $Q_{2}$ had a detrimental effect on the longitudinal behavior, and for this reason the entrance and exit angles were set to zero, $\beta_{1}=\beta_{2}=0$, while the required $Q_{2}$ was obtained by shaping only the internal part of the inflector. It is however possible that a more optimal design exists, which includes the use of $\beta_{1}$ and $\beta_{2}$.

The transfer matrices $R$ of the inflectors are shown in Tables III-V. The matrix $R$ gives the transfer function between $\left(u, u^{\prime}, h, h^{\prime}, \ell, \delta\right)_{1}$ as measured $9 \mathrm{~cm}$ upstream of the inflector, and $\left(u, u^{\prime}, h, h^{\prime}, \ell, \delta\right)_{2}$ at the first acceleration gap $6 \mathrm{~cm}$ downstream of the inflector. The unrotated coordinates $(u, h, s)$ are used here, since the final $\hat{u}$ is completely vertical, and $\hat{h}$ horizontal, making it easier to interpret the results. The starting point of the computation was chosen to be in a region where the magnetic field is negligible. This means the rotational effect on the beam, due to the growing solenoidal magnetic field when entering the cyclotron pole gap, is fully included in $R$. This effect was found to have a significant influence on the optics. The transfer matrices are symplectic, with small errors (maximum error is 0.03 ) when computing the symplectic condition $\Omega=R^{T} \Omega R$, indicating that the numerical
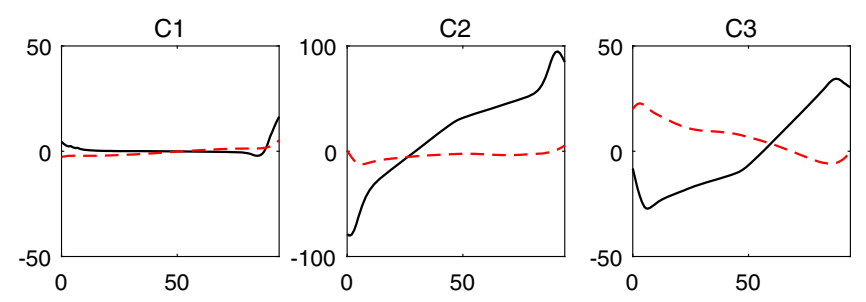

FIG. 10. Plot of the numerically measured gradient parameters $Q_{1}$ (dashed line) and $Q_{2}$ (solid line) versus the path length (in $\mathrm{mm}$ ) along the inflectors. Note that the $\mathrm{C} 2$ graph has a different vertical scale.
TABLE III. Transfer matrix $R$ of $\mathrm{C} 1(|R|=1.00)$.

\begin{tabular}{lrrrrr}
\hline \hline-1.3078 & -0.0216 & -2.1882 & -0.2905 & 0 & -0.2462 \\
-10.951 & -0.2732 & -21.255 & -3.2250 & 0 & -1.9985 \\
0.8273 & 0.0470 & -0.3371 & -0.0648 & 0 & -0.0166 \\
-2.3343 & 0.9307 & -3.7095 & -1.0723 & 0 & -1.5982 \\
1.4403 & 0.0834 & 0.3830 & 0.1273 & 1 & 0.1793 \\
-0.0027 & -0.0015 & 0.0276 & 0.0031 & 0 & 1.0016 \\
\hline \hline
\end{tabular}

TABLE IV. Transfer matrix $R$ of C2 $(|R|=1.02)$.

\begin{tabular}{lrrrrr}
\hline \hline-0.3896 & -0.1009 & -0.4583 & -0.0645 & 0 & -0.0830 \\
6.5872 & -0.3761 & -0.7189 & -0.5261 & 0 & -0.1957 \\
0.0830 & -0.0119 & 0.1719 & 0.0532 & 0 & -0.1083 \\
5.7389 & 1.4438 & -28.173 & -3.9187 & 0 & -1.1680 \\
-1.1539 & -0.1600 & 3.2106 & -0.5152 & 1 & 0.0914 \\
-0.0105 & -0.0019 & 0.0217 & 0.0020 & 0 & 1.0012 \\
\hline \hline
\end{tabular}

TABLE V. Transfer matrix $R$ of C3 $(|R|=1.00)$.

\begin{tabular}{lrrrrr}
\hline \hline-0.2892 & 0.0156 & -1.9882 & -0.2617 & 0 & -0.1862 \\
-2.0699 & -0.2359 & -11.574 & -1.9768 & 0 & -1.1793 \\
0.4382 & 0.0123 & -0.3062 & -0.0456 & 0 & -0.0555 \\
11.271 & 2.3691 & -6.1017 & -1.2424 & 0 & -1.6012 \\
0.1208 & -0.0490 & -0.3455 & 0.0546 & 1 & 0.1561 \\
0.0152 & 0.0034 & -0.0162 & -0.0017 & 0 & 0.9961 \\
\hline \hline
\end{tabular}

computations are reasonably accurate. The units of $(u, h, \ell)$ are in $\mathrm{mm}$, and $\left(u^{\prime}, h^{\prime}, \delta\right)$ are in mrad. In other words, $\delta$ is in units of $0.1 \%$ (this is required for $R$ to be symplectic).

The magnitudes of the vertical terms, $u_{2}$ and $u_{2}^{\prime}$, are described by the first two rows of $R$ and mostly follow the sequence $C 1>C 3>C 2$. This is especially the case for those values corresponding to the influence of $\left(h, h^{\prime}\right)_{1}$ on $\left(u, u^{\prime}\right)_{2}$, as well as for the dispersion terms $R_{16}$ and $R_{26}$. The values corresponding to the influence of $\left(u, u^{\prime}\right)_{1}$ on $\left(u, u^{\prime}\right)_{2}$ do not follow the same pattern, but are much smaller. The resulting effect, as shown by the phase-space plots of Fig. 9, is that $\mathrm{C} 2$ has the best vertical performance, followed by $\mathrm{C} 3$ and then $\mathrm{C} 1$.

The magnitudes of the longitudinal terms, $\ell_{2}$ and $\delta_{2}$, are described by the last two rows of $R$. The coupling between the transverse $\left(u, u^{\prime}, h, h^{\prime}\right)_{1}$ space and the longitudinal position $\ell_{2}$ is represented by the terms $R_{51}, R_{52}, R_{53}$ and $R_{54}$. The general pattern observed in the magnitude of these terms is that $C 2>C 1>C 3$. This is particularly true for $R_{51}$ where the $\mathrm{C} 3$ term is much smaller than the others, and the $R_{53}$ term where $\mathrm{C} 2$ is much greater than the others. In this fashion the $\mathrm{C} 3$-inflector design is capable of limiting the $\ell_{2}$-spread due to the transverse-longitudinal coupling.

The behavior in the longitudinal $(\ell, \delta)$-subspace is described by the $2 \times 2$ matrix in the bottom right-hand corner of $R$ : 

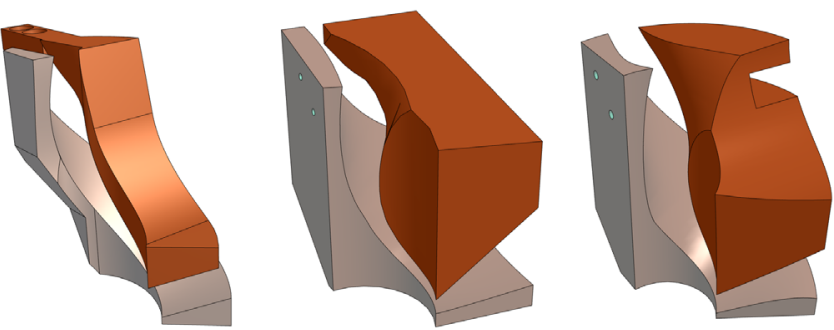

FIG. 11. 3D models of the $\mathrm{C}$-inflectors, as they were manufactured: C1 (left), C2 (middle) and C3 (right). C1 has parallel electrodes in the $\left(u_{r}, h_{r}\right)$ plane, $\mathrm{C} 2$ has straight nonparallel lines, and $\mathrm{C} 3$ has quadratic lines. For $\mathrm{C} 2$ the entrance and exit edges are not perpendicular to the central path.

$$
\left[\begin{array}{ll}
R_{55} & R_{56} \\
R_{65} & R_{66}
\end{array}\right]=\left[\begin{array}{cc}
1 & R_{56} \\
0 & 1
\end{array}\right]
$$

This matrix is equivalent to a drift space where $R_{56}$ represents the optical path length. The effect of $R_{56}$ may therefore be regarded as shifting the location of the focal point of the buncher, which will introduce an $\ell_{2}$-spread at the original focal point. This can however be compensated for by adjusting the focal length of the buncher (by tuning the buncher voltage during cyclotron operation) so that the focal point remains at its original location, and no $\ell_{2}$-spread is introduced by the inflector due to $R_{56}$.

To provide a general sense of the physical inflector shapes, 3D images of their designs are shown in Fig. 11.

\section{EXPERIMENTAL RESULTS}

The $\mathrm{C} 3$ inflector was constructed out of copper using a computer numerical control (CNC) mill, requiring 64 hours of machining time, and it was tested in the SPC2 cyclotron. Experiments were conducted to determine the transmission using all three inflectors $\mathrm{C} 1, \mathrm{C} 2$ and $\mathrm{C} 3$, where the beam current was measured throughout the cyclotron, and compared to the current on the Faraday cup immediately upstream of the cyclotron to obtain the transmission. The $\mathrm{C} 2$ and $\mathrm{C} 3$ transmission was then normalized as a fraction of the $\mathrm{C} 1$ transmission, to obtain the relative transmission which indicates how the new $\mathrm{C} 2$ and $\mathrm{C} 3$ inflectors perform compared to the original $\mathrm{C} 1$. The experimental results without the buncher activated are shown in Fig. 12. It indicates a relative transmission of about $120 \%$ for $\mathrm{C} 3$ and about $200 \%$ for C2. This is mostly due to the very good vertical injection of $\mathrm{C} 2$, but also shows that $\mathrm{C} 3$ is a vertical improvement over the original inflector.

The performance with a buncher is shown in Fig. 13. For both $\mathrm{C} 2$ and $\mathrm{C} 3$ the relative transmission within the cyclotron is roughly $150 \%$, but at the extraction this improvement largely disappears for $\mathrm{C} 2$ while it increases for $\mathrm{C} 3$. Activating the buncher decreases the relative transmission of C2 (originally 200\%), but increases that of C3 (originally 120\%). This can be explained by C2

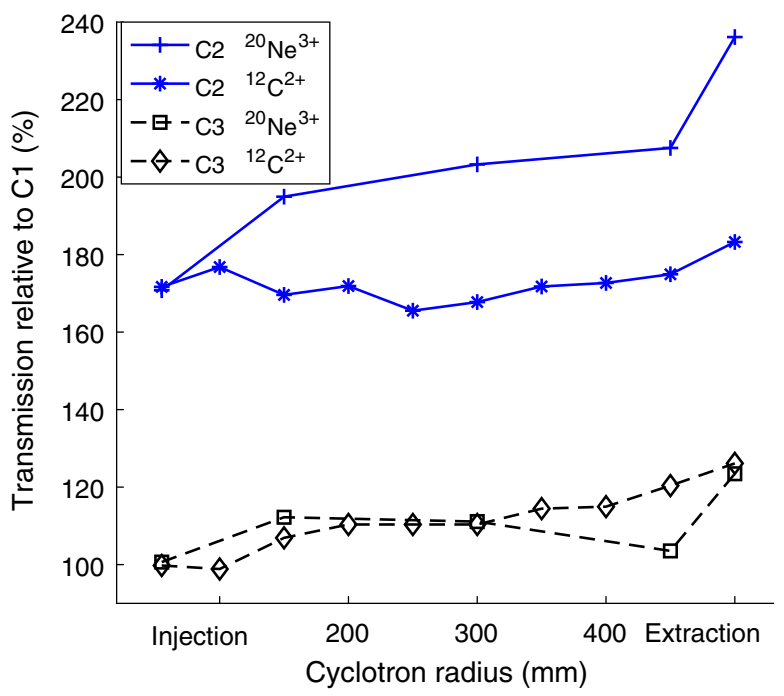

FIG. 12. The transmission of $\mathrm{C} 2$ and $\mathrm{C} 3$, as a percentage of $\mathrm{C} 1$ transmission, without a buncher. $\mathrm{C} 2$ performs very well, since it was optimized for vertical focusing only, and C3 shows a small improvement.

having worse longitudinal performance than $\mathrm{C}$, while $\mathrm{C} 3$ is an improvement on $\mathrm{C} 1$.

The good longitudinal properties of C 3 are supported by Fig. 14, where the influence of the buncher is shown. The transmission through the cyclotron is plotted as a function of the rf phase offset between the Dee voltage and the buncher voltage. When the buncher is far away from its optimal phase, the transmission is about half of the nobuncher current, which is close to the expected theoretical value for a first harmonic buncher. At the optimal phase offset, $\mathrm{C} 2$ produces an improvement in transmission of

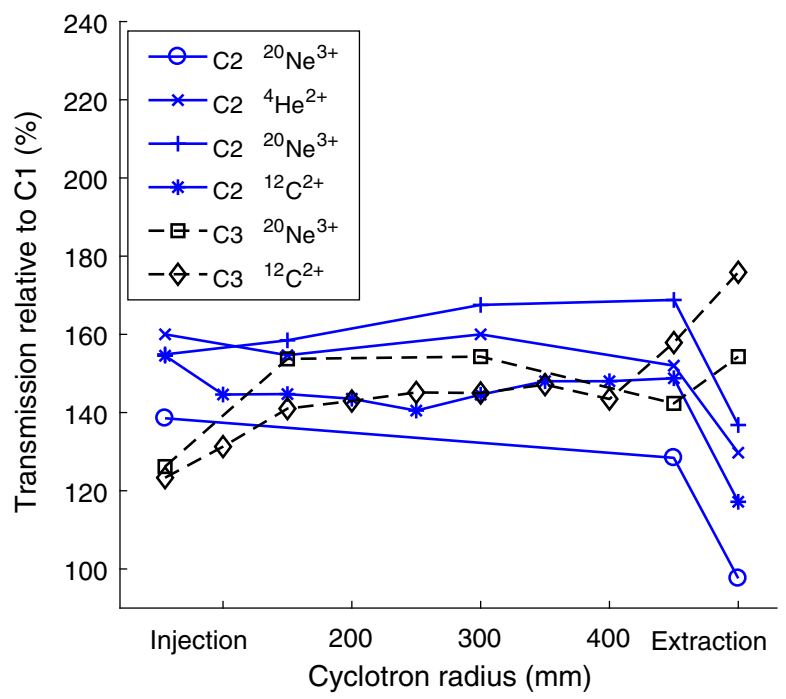

FIG. 13. The transmission of $\mathrm{C} 2$ and $\mathrm{C} 3$, as a percentage of $\mathrm{C} 1$ transmission, with the buncher active. The relative transmission of $\mathrm{C} 3$ increases when the buncher is activated, while that of $\mathrm{C} 2$ reduces. 


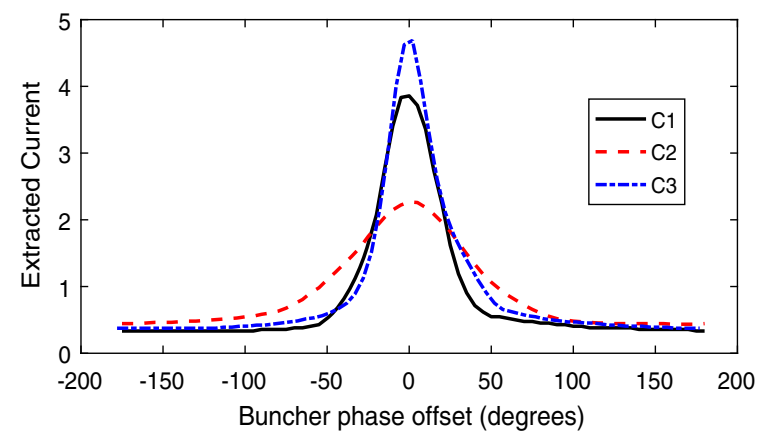

FIG. 14. Buncher efficiency: the extracted current when the buncher phase is varied, as a fraction of the no-buncher current. The phase has been normalized so that the buncher performance is optimal at zero degrees.

around 2.1 times compared to the no-buncher current, $\mathrm{C} 1$ improves by 3.8 times, while $\mathrm{C} 3$ improves the transmission by 4.8 times.

Since only two sets of independent measurements have been obtained with $\mathrm{C} 3$ so far (six sets for $\mathrm{C} 2$ ), these results should be regarded as patterns, rather than exact numerical values. From the $\mathrm{C} 2$ experiments it is however clear that all the measurements follow the overall pattern reasonably well.

\section{CONCLUSION}

The work described here shows that the spiral inflector electrode can be shaped to simultaneously achieve better longitudinal and vertical focusing, thereby substantially improving the spiral inflector performance. Along the central path the first order electric field gradients are determined by two parameters $Q_{1}(s)$ and $Q_{2}(s)$, producing quadrupole and skew-quadrupole fields in the transverse plane. These quadrupole parameters dictate the shape of the electrode surfaces and can be freely selected by the inflector designer to influence the optics.

[1] J. Belmont and J. Pabot, Study of axial injection for the Grenoble cyclotron, IEEE Trans. Nucl. Sci. 13, 191 (1966).

[2] B. Gikal, G. Gulbekian, and I. Ivanenko, Compensation of the beam vertical defocusing at the exit of U400 cyclotron spiral inflector, in Proceedings of 21st Russian Particle
Accelerators Conference (RuPAC 2008) (Joint Institute for Nuclear Research, Dubna, 2008), https://accelconf.web .cern.ch/r08/papers/TUAPH12.pdf.

[3] K. L. Brown and R. Servranckx, Cross plane coupling and its effect on projected emittance, Part. Accel. 36, 121 (1989), https://cds.cern.ch/record/200630/files/p121.pdf.

[4] I. Ivanenko, The methods of compensation of the beam vertical divergence at the exit of spiral inflector in cyclotrons, in 21st International Conference on Cyclotrons and Their Applications (Cyclotrons' 16), Zurich, Switzerland, 2016 (JACOW, Geneva, Switzerland), pp. 221-223.

[5] H. Tsutsui, Report No. JP5606793B2, 2010.

[6] A. Barnard, J. Broodryk, J. Conradie, J. De Villiers, J. Mira, F. Nemulodi, and R. Thomae, Vertical focusing with a field gradient spiral inflector, in Proceedings of the 22nd International Conference on Cyclotrons and their Applications (Cyclotrons 19), Cape Town, South Africa, 2019 (JACOW, Geneva, Switzerland, 2019), pp. 58-61.

[7] Opera-3D Suite version 17R1, TOSCA electromagnetic FEA simulation software (2019).

[8] Z. Du Toit, J. Cornell, A. Müller, J. Delsink, P. Rohwer, W. Van Heerden, J. De Villiers, D. Fourie, S. Burger, P. Celliers et al., Commissioning of the injector cyclotron for polarized and heavy ions at NAC, in Proceedings of the 14th International Cyclotron Conference and Their Applications (World Scientific Publishing, Singapore, 1996), pp. 28-31, ISBN 981022625X, http://accelconf .web.cern.ch/c95/.

[9] L. W. Root, Design of an inflector for the TRIUMF cyclotron, M.Sc. Thesis, University of British Columbia, 1972.

[10] V. Smirnov, Central region design in a compact cyclotron, Phys. Part. Nucl. Lett. 16, 34 (2019).

[11] M. Sekiguchi and Y. Shida, Orbit analysis of the spiral inflector for cyclotrons, Technical Report, Tokyo University, 1992.

[12] M. R. Spiegel, Schaum's Outline of Theory and Problems of Vector Analysis and an Introduction to Tensor Analysis (Schaum Pub. Co., New York, 1974), ISBN: 9780070602281.

[13] J. De Villiers, J. Broodryk, J. Conradie, F. Nemulodi, R. Thomae, J. Yang, and T. Zhang, Numerical orbit tracking in $3 \mathrm{D}$ through the injector cyclotron for heavy ions at iThemba LABS, in 21st International Conference on Cyclotrons and Their Applications (Cyclotrons' 16), Zurich, Switzerland (JACOW, Geneva, Switzerland, 2016), pp. 71-74. 